\title{
Correlação empírica para massa específica de biodiesel de fontes diversas e suas misturas com o diesel
}

\section{Paulo Gabriel Ferreira de Azevedo', Ivan Costa da Silva ${ }^{2}$, Cláudia Teresa Teles Farias ${ }^{2}$ e Luiz Antonio Pimentel Cavalcanti ${ }^{2}$}

${ }^{1}$ Instituto Federal de Educação, Ciência e Tecnologia da Bahia. Curso de Engenharia Química. Campus Salvador. Rua Emídio dos Santos S/N. Bairro do Barbalho. Salvador-BA, Brasil (CEP 40301-015). E-mail: paulo97745@gmail.com.

${ }^{2}$ Instituto Federal de Educação, Ciência e Tecnologia da Bahia. Campus Salvador. Rua Emídio dos Santos S/No. Bairro de Barbalho. Salvador-BA, Brasil (CEP 40301-015).

Resumo. Diante dos problemas ambientais e econômicos causados pelo uso excessivo de combustíveis de origem petrolífera, o biodiesel se mostra uma alternativa viável para uso em motores que operam no ciclo diesel. Para tal aplicação, o conhecimento das propriedades fluidodinâmicas desse combustível, como a massa específica, é indispensável, já que as mesmas estão diretamente relacionadas com o desempenho do motor. 0 presente trabalho teve por objetivo avaliar um modelo de regressão linear com o intuito de predizer a massa específica dos biodiesel de algodão (B100-A), soja (B100-S) e sebo bovino (B100-SB), bem como suas misturas com diesel petróleo (misturas BX). Tais misturas foram formuladas com base gravimétrica com percentuais de biodiesel na mistura de $5 \%$, $10 \%, 20 \%, 30 \%, 40 \%$ e $50 \%(\mathrm{~m} / \mathrm{m})$ e foram avaliadas a massa específica dos combustíveis na faixa de temperatura de 293,15 K a $363,15 \mathrm{~K}$ em intervalos de $10 \mathrm{~K}$. Com base nos dados experimentais de massa específica dos biodiesel avaliados e suas misturas com o diesel, provenientes da literatura, foram construídos gráficos tridimensionais que relacionam a massa específica em função da temperatura e fração de biodiesel presente na mistura em cada caso, verificou-se que os dados experimentais poderiam ser ajustados a um plano, dessa forma, obteve-se um modelo para a essa propriedade, sendo que os parâmetros para cada tipo de biodiesel foram estabelecidos pelo software Matlab, utilizando o aplicativo Curve Fitting. Os ajustes dos parâmetros do modelo apresentaram desvio percentual máximo de 0,15\%, no caso do B100-S, e coeficiente de determinação $\left(R^{2}\right)$ mínimo de 0,9994, também para o B100-S. Ao avaliar os dados pelo método qui-quadrado, foi observado que o modelo proposto representa satisfatoriamente os dados avaliados, podendo este ser utilizado para predizer os valores de
Recebido

$30 / 05 / 2020$

Aceito

$28 / 07 / 2020$

Disponível on line $09 / 08 / 2020$

Publicado

$31 / 08 / 2020$

Acesso aberto

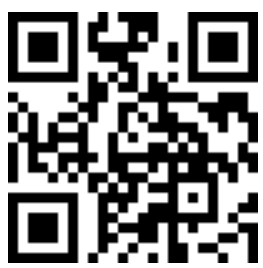

ORCID

(ㄱ) 0000-0003-0685-2928 Paulo Gabriel Ferreira de Azevedo

D 0000-0001-5990-5179 Ivan Costa da Silva

ISSN 2359-1412/RBGAS-2020-0087/2020/7/16/17/707

Rev. Bras. Gest. Amb. Sustent.

http://revista.ecogestaobrasil.net 
massa específica do biodiesel e misturas com o diesel de petróleo para o intervalo de temperatura estudado.

Palavras-chave: Biodiesel; Modelos preditivos; Massa específica.

Abstract. Empirical correlation for specific mass of biodiesel from different sources and their mixtures with diesel. In view of the economics and environmental issues caused by the excessive use of fossil fuels, biodiesel proves to be a viable alternative for applications in diesel engines. For this end, the knowledge of the fluid dynamic properties of this fuel, such as the specific mass, is essential, since they are directly related to the engine's performance. This work aimed to evaluate a linear regression model in order to predict the specific mass of biodiesel from cotton (B100-A), soy (B100-S) and beef tallow (B100-SB), as well as their mixtures with diesel oil (BX mixtures). Such mixtures were formulated on a gravimetric basis, with percentages of biodiesel in the blends of $5 \%, 10 \%, 20 \%, 30 \%$, $40 \%$ and $50 \%(\mathrm{~m} / \mathrm{m})$ and the specific mass of fuels in the temperature range of $293.15 \mathrm{~K}$ to $363.15 \mathrm{~K}$ were evaluated, at $10 \mathrm{~K}$ intervals. Based on experimental data of specific mass, from the literature, of the mentioned types of biodiesel, threedimensional graphs were plotted relating specific mass as a function of temperature and fraction of biodiesel present in the mixture, it was noted that the empirical data could be adjusted in a plane, a model was fitted for this property, and the parameters were calculated in the Matlab software, using the Curve Fitting application. The model parameters fitted showed a maximum relative error and the minimum determination coefficient $\left(\mathrm{R}^{2}\right)$ of $0.15 \%$ and 0.994 , respectively, both for B100-S. Evaluating the results by the Chi-square method, it was observed that the proposed model represents satisfactorily the experimental datas, being able to predict the specific mass values of biodiesel and their blend with diesel, in studied temperature intervals.

Keywords: Biodiesel; Predictive models; Specific weight.
다 $0000-0002-3976-613 X$

Cláudia Teresa Teles

Farias

(1) 0000-0003-4932-9387

Luiz Antonio Pimentel

Cavalcanti

\section{Introdução}

Os prejuízos da dependência demasiada de reservas energéticas oriundas do petróleo têm tomado destaque tanto no setor econômico, com o esgotamento acelerado dessas reservas gerando o aumento no preço dos combustíveis fósseis, como no ambiental, com a atenção ao aquecimento global e poluição atmosférica (RamirezVerduzco, 2013; Martins et al., 2018). Diante desse cenário, os biocombustíveis vêm se destacando, devido a seus diversos benefícios para o meio ambiente, como baixa toxicidade, renovabilidade e baixa emissão de gases de efeito estufa em sua combustão (Gülün e Bilgin, 2017).

Destaque entre os biocombustíveis, o biodiesel é definido como uma mistura de ésteres alquílicos de ácidos graxos, obtidos, mais comumente, pela reação de transesterificação alcalina por rota metílica de triglicerídeos de biomassa vegetal, animal, óleos residuais e/ou microalgas (Cavalcanti, 2013). 0 biodiesel é completamente miscível 
em diesel de petróleo, de modo que o emprego de misturas biodiesel/diesel (misturas BX) é amplamente utilizada mundialmente (Cavalcanti, 2013; Kanaveli et al., 2017).

0 conhecimento das propriedades do biodiesel em intervalos de temperatura e fração de biodiesel presente na mistura é importante nas simulações de processo de produção de biodiesel, bem como nos modelos para projeto de sistema de ignição do motor (Prieto et al., 2015). Não é viável a medição dessas propriedades em cada proporção de mistura e temperatura do processo em estudo, diante da dificuldade de análise e do custo; portanto, a utilização de modelos para predizer essas propriedades é de extrema relevância (Cavalcanti, 2016).

A massa específica é uma das propriedades físicas mais importantes dos combustíveis. A entrada do combustível na câmara de combustão é dada em base volumétrica, portanto a massa específica está diretamente relacionada com a quantidade de combustível disponível para a combustão e, dessa forma, também relacionada com o desempenho do motor. Nessa perspectiva um excesso de volume de combustível na câmara pode levar à combustão incompleta, aumentando assim as emissões de gases poluentes e de efeito estufa. Além disso, dados de massa específica dos combustíveis também são usados em projetos de reatores, torres de destilação, tanques de armazenamento e tubulações (Ramirez-Verduzco, 2013).

Diante do exposto, esse estudo visa a avaliar um modelo de regressão linear para predizer a massa específica dos biodiesel de algodão (B100-A), de soja (B100-S) e de sebo bovino (B100-SB) e suas misturas BX com o diesel em diferentes temperaturas.

\section{Fundamentação teórica}

A massa específica do biodiesel é influenciada por diversos fatores, como a temperatura, pressão, composição do combustível, rotas de síntese e composição do óleo utilizado na produção do combustível. Então, modelos que levam esses fatores em consideração são relevantes na predição dessa propriedade fluidodinâmica. 0 modelo de Liew (Equação 1) é usado para avaliar a relação entre a massa específica do biodiesel, e suas misturas BX, com a temperatura (Cavalcanti, 2016).

$$
\rho(T)=a_{1}+a_{2} T
$$

Sendo $\rho$ a massa específica em $\mathrm{kg} \cdot \mathrm{m}^{-3}$, $\mathrm{T}$ a temperatura em $\mathrm{K}$ e $\mathrm{a}_{\mathrm{i}}$ os parâmetros do modelo. Nota-se que o modelo da Equação 1 estabelece uma relação linear entre a massa específica e a temperatura. Como a relação entre esses parâmetros é inversa, espera-se que o termo $a_{2}$ da Equação 1 seja negativo.

Pham et al. (2018) estudaram os biodiesel oriundos de óleo residual, de pinhão manso e de coco (B100), suas misturas B5, B10, B20, B40, B50, B60 e B75, e o diesel, no intervalo de temperatura de 30 a $100{ }^{\circ} \mathrm{C}$. Os autores avaliaram o modelo de Liew para os dados de massa específica dos B100 e encontraram erro máximo de 0,4026\%, para o biodiesel de óleo residual, e coeficiente de correlação $\left(R^{2}\right)$ de 0,9979 . Ficou evidenciado que o modelo de Liew pode ser usado para relacionar a massa específica do biodiesel com a temperatura.

Além disso, a composição do combustível é um importante fator na modelagem dessa propriedade fluidodinâmica e precisa ser levada em consideração. Em função disso, Ramírez-Verduzco (2013) compilou dados de massa específica dos ésteres metílicos de ácidos graxos (EMAG) que compõem o biodiesel, provenientes da literatura e em distintos 
intervalos de temperatura, e examinou o modelo da Equação 2, com o objetivo de verificar a capacidade preditiva do mesmo.

$$
\rho(M, N, T)=a_{1}+\frac{a_{2}}{M}+a_{3} \cdot N+a_{4} \cdot T
$$

Sendo $\mathrm{M}$ a massa molar dos EMAG que compõem o combustível em $\mathrm{g} \cdot \mathrm{mol}^{-1}$, $\mathrm{N}$ o número de ligações duplas na cadeia principal de EMAG e T a temperatura em K. Nota-se que os termos $\mathrm{M}$ e $\mathrm{N}$ estão estritamente atrelados à constituição química do combustível.

Utilizando a Equação 2 para cada EMAG, o autor calculou a massa específica dos B100 (biodiesel produzidos do óleo de coco, canola, algodão, babaçu e soja) através da Regra de Kay (Equação 3).

$$
\rho=\sum\left(w_{i} \cdot \rho_{i}\right)
$$

Sendo $w_{\mathrm{i}}$ e $\rho_{\mathrm{i}}$ a fração mássica e a massa específica do EMAG. Em nenhum caso analisado foi constatado erro igual ou acima de $1 \%$, sendo que o erro médio foi de $0,48 \%$. Com base nisso, o autor concluiu que o modelo da Equação 2, aliado a Equação 3, reproduz razoavelmente bem as informações experimentais de massa específica dos biodiesel analisados.

Além da sua composição química, a fração do biodiesel nas misturas BX também é um fator relevante no desenvolvimento de modelos para predição da massa específica. Gabriel et al. (2020) estudaram um modelo tridimensional para predição da massa específica do biodiesel, e misturas BX, provenientes do óleo de chichá, por diferentes rotas de síntese. Os dados empíricos foram estabelecidos com variação de temperatura de 283,15 a 313,15 K, com intervalos de $5 \mathrm{~K}$, e as misturas estudadas foram B10, B15, B20, B40, B60 e B80. 0 modelo investigado pelos autores (Equação 4) leva em consideração a influência linear da temperatura, tal como o modelo de Liew (Equação 1), e o fator referente a fração de biodiesel nas misturas BX.

$$
\rho(T, X)=a_{1}+a_{2} \cdot T+a_{3} . X
$$

Onde X é a porcentagem de biodiesel presente na mistura com o diesel (mistura BX). Observando a Equação 4, nota-se que os autores atribuíram uma relação linear entre a fração de biodiesel na mistura BX e a massa específica da mistura. Neste trabalho foi encontrado erro máximo de $0,22 \%$ e $\mathrm{R}^{2}$ mínimo de 0,9936 . Com isso, tem-se que o modelo da Equação 4 representa satisfatoriamente os dados experimentais de massa específica do biodiesel, no intervalo de temperatura estudado.

\section{Materiais e métodos}

Foram utilizados dados empíricos de massa específica, provenientes da literatura, dos biodiesel de algodão, soja e sebo bovino (B100), o diesel (B0) e as misturas B5, B10, B20, B30, B40, B50, com variações de temperatura de 293 a $363 \mathrm{~K}$, com intervalos de $10 \mathrm{~K}$ (Cavalcanti, 2013). Esses dados experimentais foram tratados via regressão linear utilizando o software Matlab, com o aplicativo Curve Fitting, com o intuito de obtenção dos 
parâmetros do modelo. Foi analisado o coeficiente de determinação $\left(\mathrm{R}^{2}\right)$, obtido pelo software, e o desvio percentual médio (DPM) (Equação 5).

$$
D P M=\frac{100}{N} \sum_{i=1}^{N}\left|\frac{\rho_{\text {exp }}-\rho_{\text {pred }}}{\rho_{\text {exp }}}\right|
$$

sendo $\rho_{\text {exp }}$ e $\rho_{\text {pred }}$ os valores de massa específica medidos experimentalmente e preditos pelos modelos, respectivamente, e $\mathrm{N}$ o número de pontos experimentais; dessa forma, quanto menor o valor de DPM, mais próximos estarão os valores modelados dos dados empíricos.

A validação estatística dos modelos encontrados foi avaliada pelo teste de aderência de modelos (qui-quidrado) (Equação 6). 0 teste do qui-quadrado ( $\mathrm{X}^{2}$ ) é uma forma de avaliar se um conjunto de dados, divididos em k classes, enquadram-se em uma determinada hipótese, que nesse trabalho foram os modelos de massa específica propostos. Para tal, faz-se o uso da Equação 4, de modo a se consultar se o valor obtido por essa equação $\left(\mathrm{X}^{2}\right)$, assumindo (k-1) graus de liberdade, corrobora para aceitação ou recusa da hipótese nula, tendo uma determinada probabilidade de ocorrência.

$$
X^{2}=\sum_{i=1}^{N}\left|\frac{\left(\rho_{\text {exp }}-\rho_{\text {pred }}\right)^{2}}{\rho_{\exp }}\right|
$$

Quanto menor o valor de $\mathrm{X}^{2}$, mais aceitável a hipótese feita para um determinado conjunto de dados, isto é, o modelo representa satisfatoriamente os dados experimentais, aceitando-se um determinado nível de confiança, que neste trabalho foi de 95\%.

\section{Resultados e discussão}

Plotando-se os dados de massa específica em função da composição do biodiesel presente na mistura BX e temperatura, verifica-se um comportamento linear dos dados, tal comportamento sugere que modelo proposto pode representado pela equação de um plano que pode ajustado a Equação 4. Os parâmetros a1, a2 e a3 do modelo são exibidos na Tabela 1. Esses valores foram obtidos via regressão linear dos dados experimentais para cada biodiesel avaliado.

\begin{tabular}{|c|c|c|c|c|}
\hline Parâmetro & $a_{1}\left(\mathrm{~kg} \cdot \mathrm{m}^{-3}\right)$ & $a_{2}\left(\mathrm{~kg} \cdot \mathrm{m}^{-3} \mathrm{~K}^{-1}\right)$ & $a_{3}\left(\mathrm{~kg} \cdot \mathrm{m}^{-3}\right)$ & $\mathbf{R}^{2}$ \\
\hline Algodão & 1045 & $-0,7114$ & 45,63 & 0,9999 \\
\hline Soja & 1046 & $-0,7131$ & 42,45 & 0,9994 \\
\hline Sebo bovino & 1046 & $-0,7123$ & 33,47 & 0,9999 \\
\hline
\end{tabular}

Tabela 1. Valores ajustados dos parâmetros $a_{\mathrm{i}}$ do modelo da Equação 10.

Observando a Tabela 1, nota-se que os valores de $a_{2}$ são negativos em todos os casos, concluindo que a relação entre massa específica e temperatura é inversamente proporcional. 0 contrário é observado na relação entre massa específica e a fração de biodiesel presente na mistura BX: quanto maior a fração de biodiesel, maior o valor de massa específica. Nota-se, ainda, que os valores dos termos a $a_{2}$ são próximos, independente 
do tipo de biodiesel. Essa observação indica que nos três casos estudados, a massa específica varia de modo similar com a temperatura.

0 valor do termo $\mathrm{a}_{3}$ do biodiesel de sebo bovino é menor quando comparado com os biodiesel de algodão e de soja, tal diferença pode ser atribuída à composição de ésteres metílicos de ácidos graxos saturados, que é maior para o biodiesel de sebo bovino $(55,0 \%)$, do que para os de algodão $(26,2 \%)$ e soja $(17,27 \%)$ (Cavalcanti, 2013). 0 coeficiente de correlação $\left(\mathrm{R}^{2}\right)$ em todos os casos estão acima de 0,999 .

As Figuras 1 a 3 apresentam os dados empíricos do trabalho de Cavalcanti (2013) para massa específica dos biodiesel de algodão, soja e sebo bovino em função do percentual de biodiesel presente na mistura BX e da temperatura, bem como os planos que representam o modelo proposto pela Equação 4.

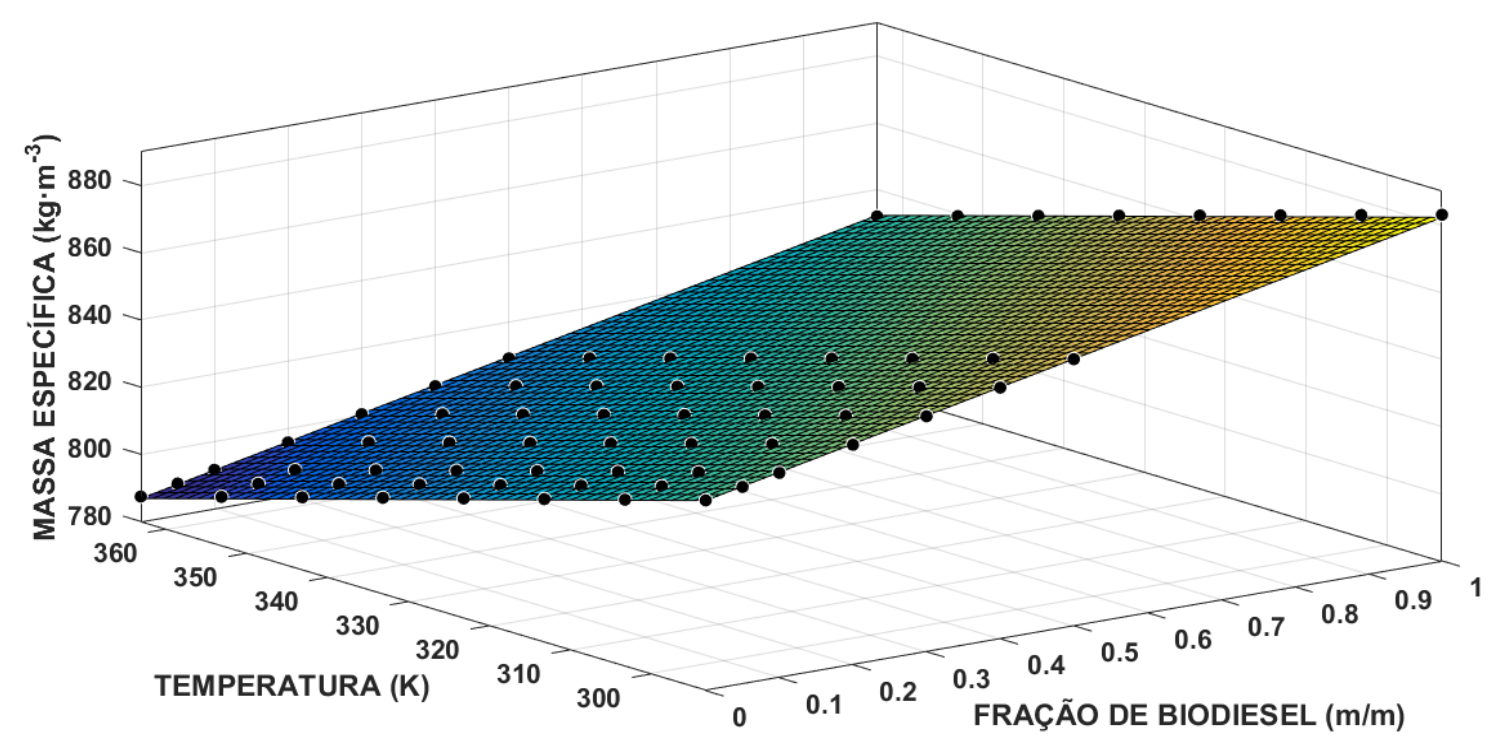

Figura 1. Superfície modelada e dados empíricos de massa específica para a mistura BX-A.

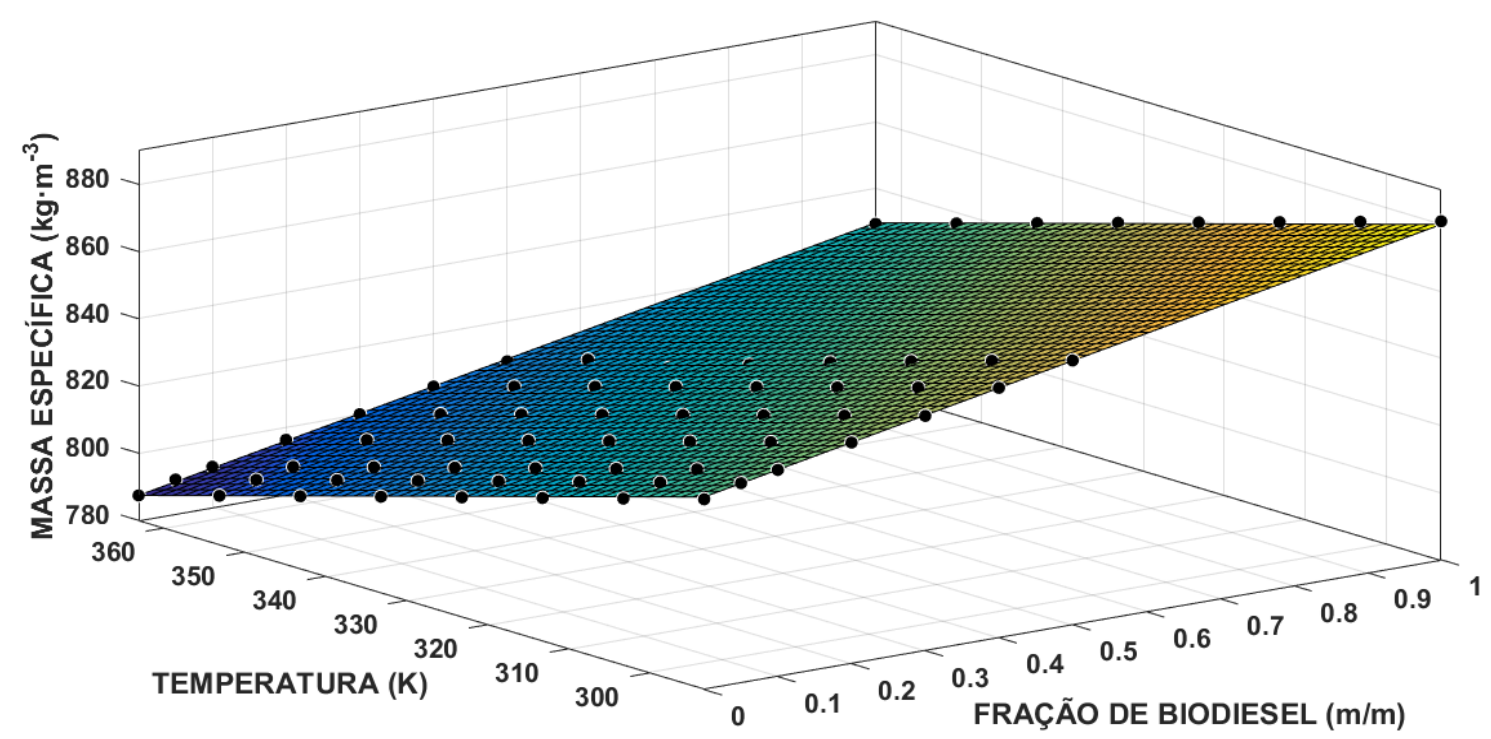

Figura 2. Superfície modelada e dados empíricos de massa específica para a mistura BX-S. 


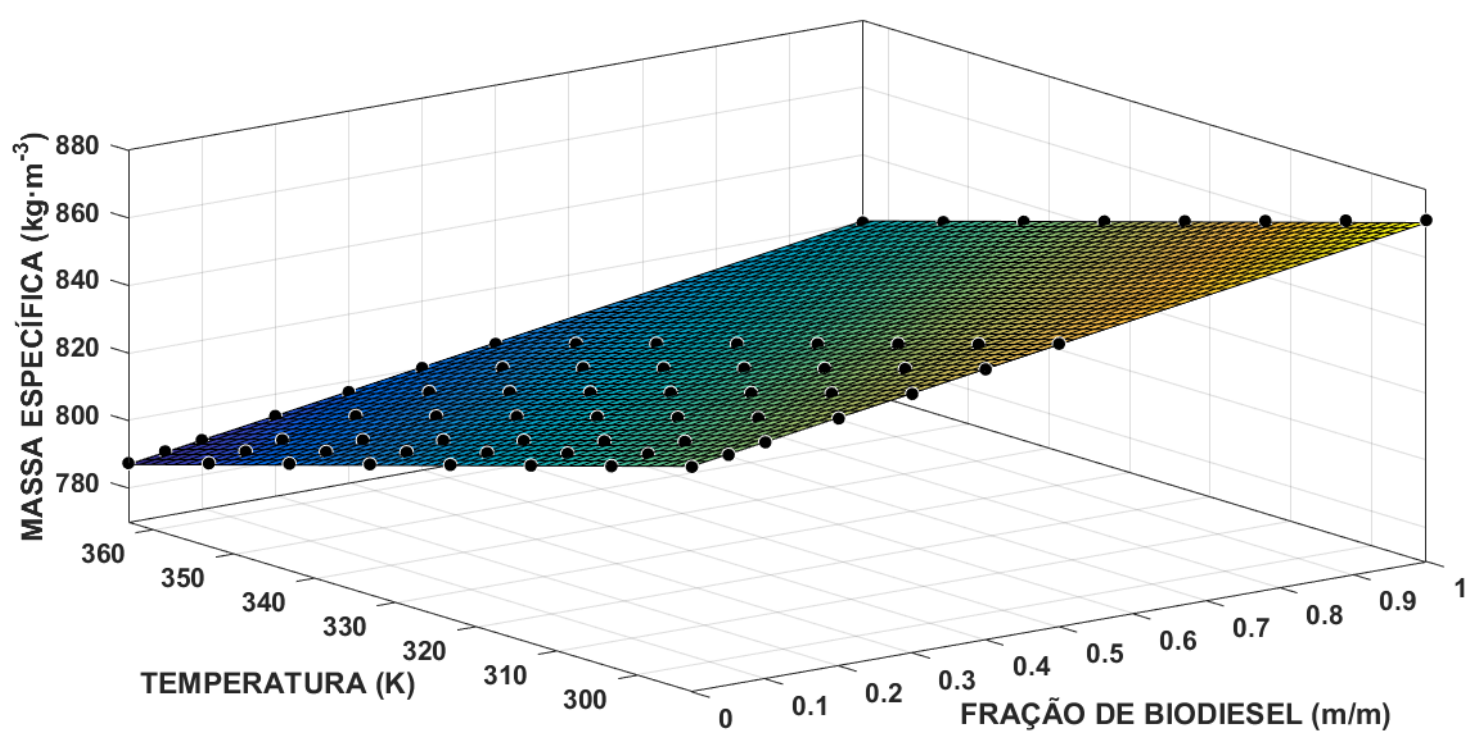

Figura 3. Superfície modelada e dados empíricos de massa específica para a mistura BX-SB

Os valores de DPM para os três tipos de biodiesel estudados estão exibidos na Tabela 2. 0 maior valor de DPM é 0,0637\%, para o biodiesel de soja. Gabriel et al. (2020) estudaram modelos tridimensionais para predição de massa específica do biodiesel, e misturas BX, provenientes do óleo de chichá, por diferentes rotas de síntese; os mesmos encontraram erros máximos de $0,22 \%$, superior aos obtidos pelo presente trabalho. Gülüm e Bilgin (2017) encontraram erro médio de 0,0607\% estudando o modelo bidimensional da Equação 1. Portanto, comparando com a literatura, os valores de DPM gerados pelo modelo da Equação 4 são satisfatórios.

Tabela 2. Valores de DPM gerados pelo modelo da Equação 4.

\begin{tabular}{|l|c|}
\hline Tipo de Biodiesel & DPM (\%) \\
\hline Algodão & 0,0244 \\
\hline Soja & 0,0637 \\
\hline Sebo bovino & 0,0467 \\
\hline
\end{tabular}

Os valores de qui-quadrado calculados $\left(\mathrm{X}^{2}\right.$ calculado $)$ pela Equação 6, com base nos dados experimentais dos três tipos de biodiesel, são mostrados na Tabela 3.

Tabela 3. Valores de $\mathrm{X}^{2}$ calculado do modelo da Equação 4 para os três tipos de biodiesel.

\begin{tabular}{|l|c|}
\hline Tipo de Biodiesel & $\mathbf{X}^{2}$ calculado \\
\hline Algodão & 0,0073 \\
\hline Soja & 0,1692 \\
\hline Sebo bovino & 0,0140 \\
\hline
\end{tabular}


Os dados experimentais foram distribuídos em 8 classes, de modo que o grau de liberdade é 7, e foi assumido o nível de confiança de $95 \%$, portanto tem-se que o valor de qui-quadrado tabelado $\left(\mathrm{X}^{2}\right.$ tabelado $)$ é de 14,067, para os três casos estudados. Em todos os casos, o $\mathrm{X}^{2}$ calculado $<\mathrm{X}^{2}$ tabelado, evidenciando que o modelo da Equação 4 se adequa bem aos dados experimentais, em todos os casos estudados.

Para avaliar o modelo da Equação 4, foram analisados dados experimentais não incluídos nas regressões lineares dos dados de massa específica dos misturas BX-A, BX-S e BX-SB. Nessas regressões, foram utilizados dados no intervalo de temperatura de 293,15 a $363,15 \mathrm{~K}$, com intervalos de $10 \mathrm{~K}$. Outra forma de validação do modelo é propor a utilização de dados experimentais de massa específica dos três tipos de biodiesel para $\mathrm{T}=$ 283,15 K e aplicando esse valor de temperatura a Equação 4, foram obtidos gráficos das Figuras de 4 a 6 , que contém a reta modelada e os dados experimentais.

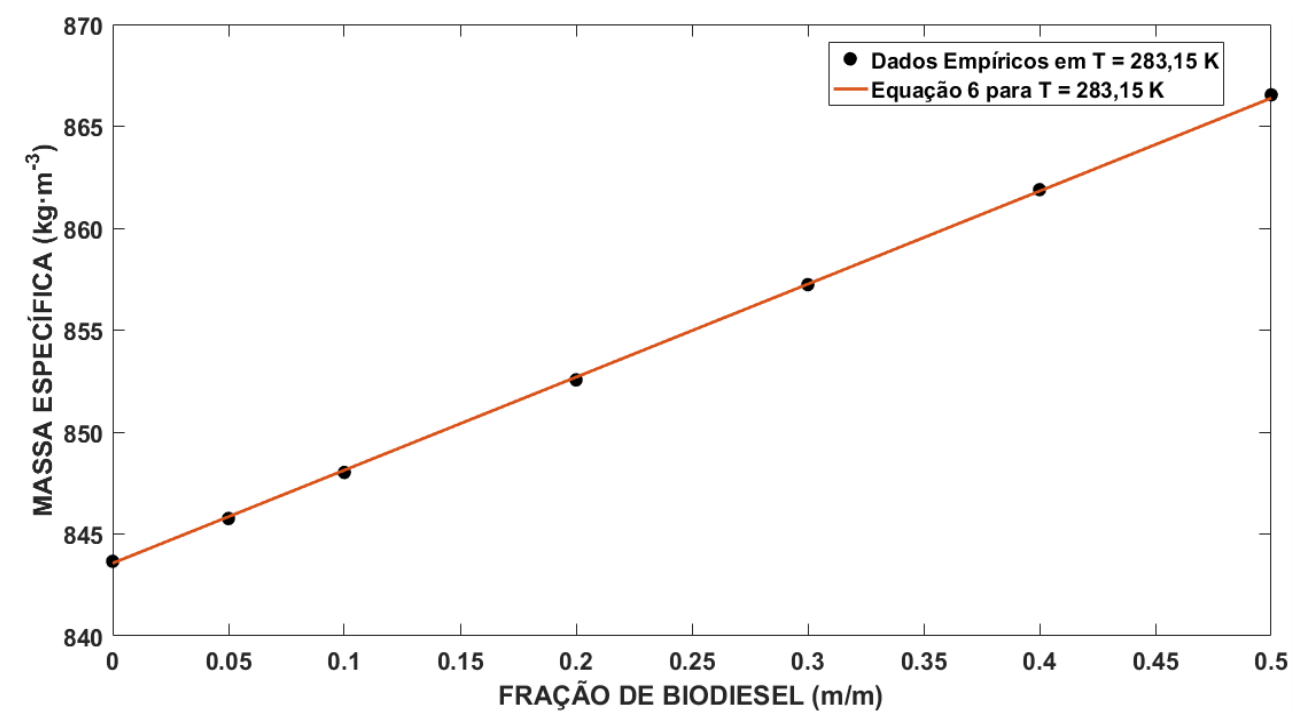

Figura 4. Reta modelada e dados experimentais da mistura BX-A para $\mathrm{T}=283,15 \mathrm{~K}$.

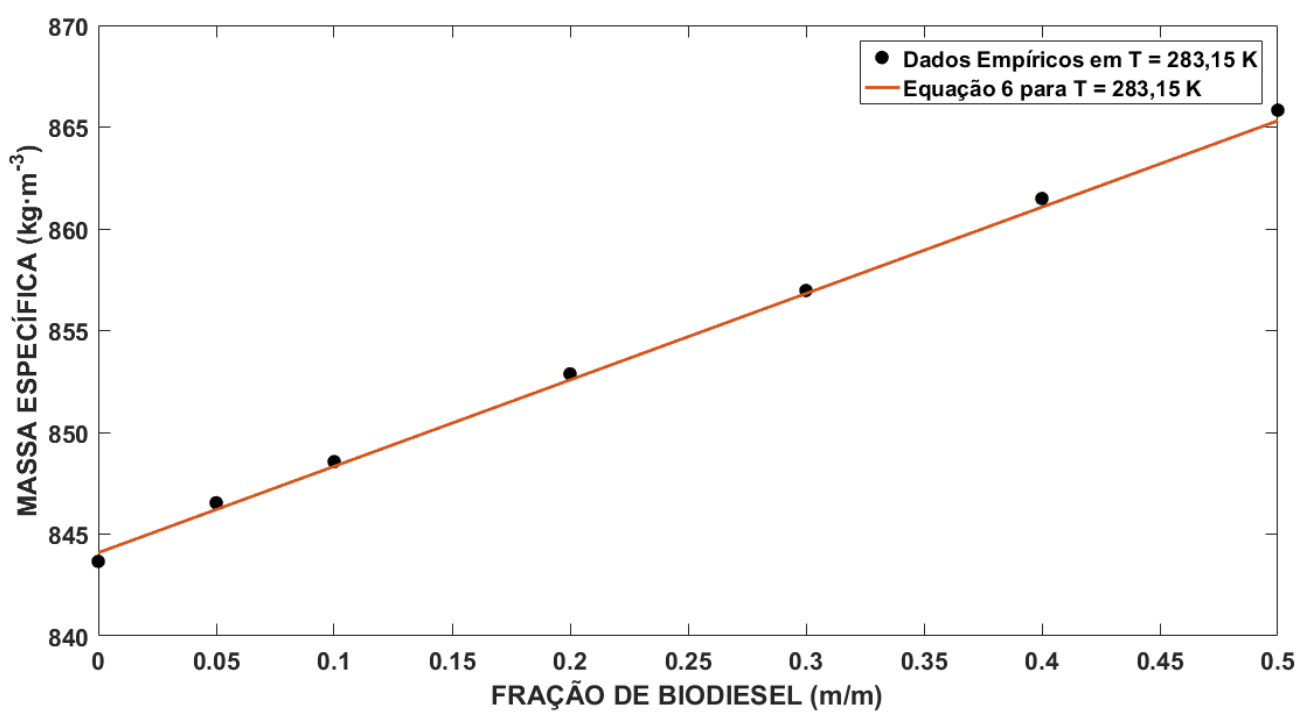

Figura 5. Reta modelada e dados experimentais da mistura BX-S para $\mathrm{T}=283,15 \mathrm{~K}$ 


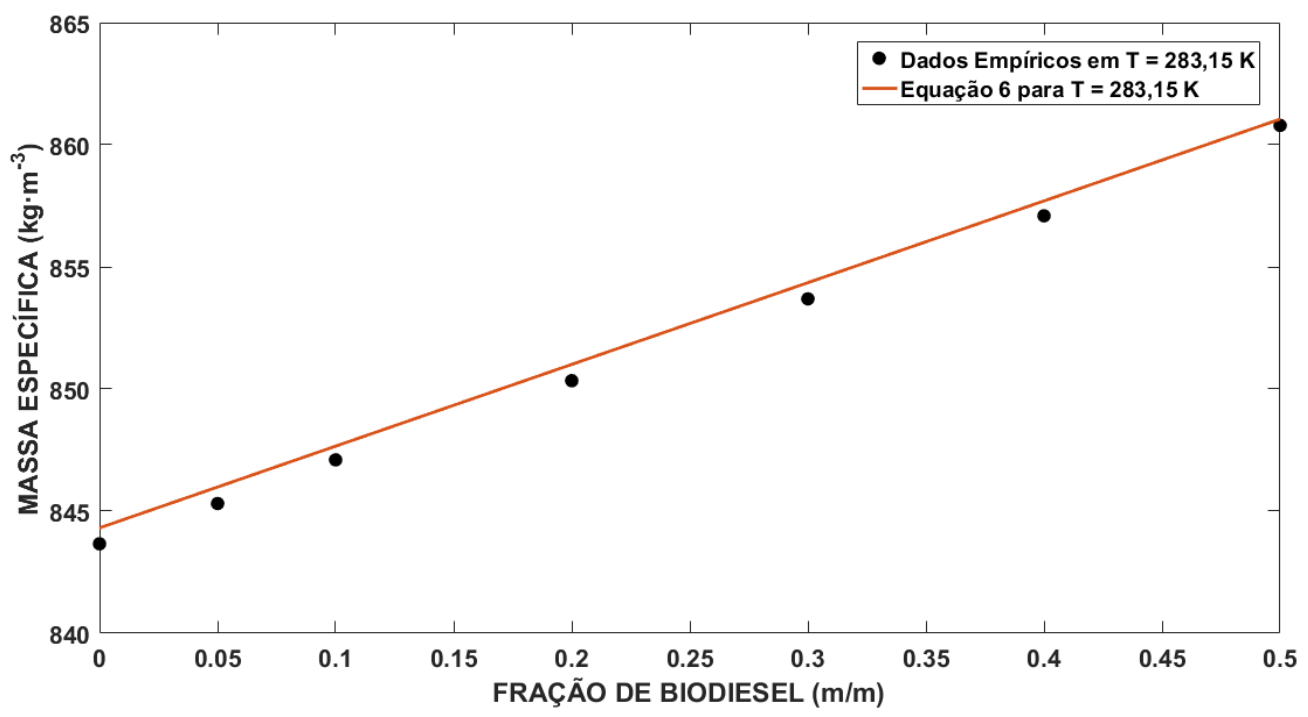

Figura 6. Reta modelada e dados experimentais da mistura BX-SB para $\mathrm{T}=283,15 \mathrm{~K}$.

Observando as Figuras de 4 a 6, nota-se que somente no caso da mistura BX-SB os pontos experimentais não estão contidos na reta modelada. Os valores de DPM gerados pela reta formada e os dados experimentais, ambos para $\mathrm{T}=283,15 \mathrm{~K}$, foram calculados $\mathrm{e}$ se apresentam na Tabela 4.

Tabela 4. Valores de DPM gerados pela Equação 6 e dados empíricos para T = 283,15 K.

\begin{tabular}{|l|c|}
\hline Tipo de Biodiesel & DPM (\%) \\
\hline Algodão & 0,0114 \\
\hline Soja & 0,0397 \\
\hline Sebo bovino & 0,0686 \\
\hline
\end{tabular}

O valor máximo de DPM exibido na Tabela 4 é de 0,0686\%, para o BX-SB, o que condiz com a análise visual do gráfico da Figura 6. Deste modo, constata-se que os valores modelados pela Equação 4 conformam-se adequadamente aos dados experimentais, ambos para $\mathrm{T}=283,15 \mathrm{~K}$.

\section{Conclusão}

Os dados experimentais de massa específica das misturas BX-A, BX-S e BX-SB, no intervalo de temperatura estudado, adequaram-se satisfatoriamente a um modelo tridimensional planar. A aplicação da regressão linear nos dados empíricos estabeleceu parâmetros com $\mathrm{R}^{2}$ próximos a 1 e gerou superfícies que representa a massa específica em função da temperatura e da fração de biodiesel presente na mistura. 0 modelo gerou pequenos desvios entre os valores modelados e os empíricos, mas os dados de DPM indicaram que essa diferença é aceitável e próximo dos encontrados nos resultados de trabalhos similares. Com o teste do qui-quadrado, foi concluído que o modelo representa adequadamente os dados experimentais. Diante do conjunto de resultados, conclui-se que o modelo estudado pode ser usado para predizer a massa específica dos biodiesel de 
algodão, soja e sebo bovino e suas misturas BX, no intervalo de temperatura avaliado, com aplicações em sistemas de modelagem de combustão, em motores, ou em equipamentos de operações unitárias, como reatores.

\section{Conflito de interesses}

Os autores declaram não haver conflito de interesses.

\section{Referências}

Cavalcanti, L. A. P. Modelos de regressão aplicados para predição das propriedades físicas das misturas do biodiesel de sebo bovino com o diesel. CIATEC-UPF, v. 8, n. 2, p. 22-33, 2016.

Cavalcanti, L. A. P. Reologia e melhoramento das propriedades de escoamento a frio de biodiesel e suas misturas BX. Recife: Universidade Federal de Pernambuco, 2013. (Tese de doutorado).

Gabriel, R.; Vieira, W. T.; Soletti, J. I.; Ribeiro, L. M. O.; Carvalho, S. H. V. Empirical modeling of different viscosity and density behavior of biodiesel from chichá (Sterculia striata) with diesel versus temperature variation. Journal of King Saud University - Science, v. 32, n. 1, p. 628-635, 2020. https://doi.org/10.1016/j.jksus.2018.08.009

Gülüm, M.; Bilgin, A. Measurements and empirical correlations in predicting biodieseldiesel blends' viscosity and density. Fuel, v.199, p.567-577, 2017. https://doi.org/10.1016/j.fuel.2017.03.001

Kavaneli, I. P.; Atzemi, M.; Lois, E. Predicting the viscosity of diesel/biodiesel blends. Fuel, v. 199, p. 248-263, 2017. https://doi.org/10.1016/j.fuel.2017.02.077

Martins, F.; Felgueiras, C.; Smitková, M. Fossil fuel energy consumption in European countries. Energy Procedia, v. 153, p. 107-111, 2018. https://doi.org/10.1016/j.egypro. 2018.10.050

Pham, M. T.; Hoang, A. T.; Le, A. T.; Tawaha, A. R. M. S. A.; Dong, V. H.; Le, V. V. Measurements and prediction of the density and viscosity of biodiesel blends. International Journal of Technology, v. 5, p. 1015-1026, 2018.

Prieto, N. M. C. T.; Ferreira, A. G. M.; Portugal, A. T. G.; Moreira, R. J.; Santos, J. B. Correlation and prediction of biodiesel density for extended ranges of temperature and pressure. Fuel, v. 141, p. 23-38, 2015. https://doi.org/10.1016/j.fuel.2014.09.113

Ramirez-Verduzco, L. F. Density and viscosity of biodiesel as a function of temperature: Empirical models. Renewable and Sustainable Energy Reviews, v. 19, p. 652-665, 2013. https://doi.org/10.1016/j.rser.2012.11.022 